\title{
GREEN'S FUNCTIONS AND RIEMANN'S METHOD
}

\author{
by A. G. MACKIE \\ (Received 5th October 1964)
}

\section{The role of the Green's function}

Methods for solving boundary value problems in linear, second order, partial differential equations in two variables tend to be somewhat rigidly partitioned in some of the standard text-books. Problems for elliptic equations are sometimes solved by finding the fundamental solution which is defined as a solution with a given singularity at a certain point. Another approach is by way of Green's functions which are usually defined as solutions of the original homogeneous equations now made inhomogeneous by the introduction of a delta function on the right hand side. The Green's function coincides with the fundamental solution for elliptic equations but exhibits a totally different type of singularity for parabolic or hyperbolic equations. Boundary value problems for hyperbolic equations can often by solved by Riemann's method which depends on the existence of an auxiliary function called the Riemann or sometimes the Riemann-Green function. The main object of this paper is to show the close relationship between Riemann's method and the method of Green's functions. This not only serves to unify different methods of solution of boundary value problems but also provides an additional method of determining Riemann functions for given hyperbolic equations. Before establishing these relationships we shall survey the general approach to boundary value problems through the use of the Green's function.

We consider a linear differential operator $L$ in the two variables $x$ and $y$ and the equation

$$
L[\phi]=F(x, y) .
$$

A Green's function $G\left(x, y ; x_{0}, y_{0}\right)$ for the operator $L$ is defined as a solution of

$$
L[G]=\delta\left(x-x_{0}\right) \delta\left(y-y_{0}\right),
$$

where the delta function on the right hand side vanishes except when $x=x_{0}$ and $y=y_{0}$ although the double integral of this function over any region including $\left(x_{0}, y_{0}\right)$ as an internal point is unity. The Green's function for a given boundary value problem is now defined as a function which satisfies (1.2) together with certain homogeneous boundary conditions on a given curve. Clearly any two Green's functions differ by a solution of the homogeneous equation $L[\phi]=0$.

A few examples will help to clarify this definition. When $L$ is the Laplacian 
operator and the boundary conditions are $\phi=0$ on $y=0, \phi \rightarrow 0$ at infinity in the upper half-plane, then

$$
G\left(x, y ; x_{0}, y_{0}\right)=\frac{1}{2 \pi} \log \frac{\left(x-x_{0}\right)^{2}+\left(y-y_{0}\right)^{2}}{\left(x-x_{0}\right)^{2}+\left(y+y_{0}\right)^{2}} .
$$

For the wave equation

$$
\frac{\partial^{2} \phi}{\partial x^{2}}-\frac{\partial^{2} \phi}{\partial y^{2}}=0
$$

with associated boundary conditions $\phi=\partial \phi / \partial y=0$ on $y=0$,

$$
\begin{aligned}
G\left(x, y ; x_{0}, y_{0}\right) & =-\frac{1}{2} & & \left(\left|x-x_{0}\right|<y-y_{0}\right), \\
& =0 & & \left(\left|x-x_{0}\right|>y-y_{0}\right) .
\end{aligned}
$$

Graphically this means that $G$ is zero except in the right angle formed by the forward-facing characteristics $\left(x_{0}, y_{0}\right)$ where it is $-\frac{1}{2}$. For the diffusion equation

$$
\frac{\partial^{2} \phi}{\partial x^{2}}-\frac{\partial \phi}{\partial y}=0
$$

with associated boundary condition $\phi=0$ on $y=0$,

$$
G\left(x, y ; x_{0}, y_{0}\right)=-\frac{H\left(y-y_{0}\right)}{2 \pi^{\frac{1}{2}}\left(y-y_{0}\right)^{\frac{1}{2}}} e^{-\left(x-x_{0}\right)^{2} / 4\left(y-y_{0}\right)},
$$

where $H$ denotes the Heaviside or unit function.

Each of these Green's functions has a simple physical interpretation. Equation (1.3) is the electrostatic potential in a half-plane due to a charge $-1 / 2 \pi$ at $\left(x_{0}, y_{0}\right)$ when the boundary $y=0$ is earthed. If $y$ is interpreted as the time co-ordinate in (1.4) and (1.5), (1.4) is the displacement of an infinite string, initially at rest in equilibrium, when it is struck a blow at $x=x_{0}$ at time $y=y_{0}$ and (1.5) the distribution of temperature in a uniform infinite bar, initially at zero temperature, subject to a heat source applied instantaneously when $y=y_{0}$ at the position $x=x_{0}$. A point of interest to note is that the singularities are quite different in the three cases. In (1.3), $G$ is logarithmically infinite at $\left(x_{0}, y_{0}\right)$ but is regular all over the half-plane $y>0$ apart from this point. In (1.4), $G$ is finite everywhere and the singularity now takes the form of a discontinuity which persists along the forward-facing characteristics through $\left(x_{0}, y_{0}\right)$. Finally in (1.5), $G$ has a more elaborate type of singularity at $\left(x_{0}, y_{0}\right)$ than in (1.3) and whether $G$ is infinite at this point or not depends on the direction of approach. For any $x \neq x_{0}, G$ as a function of $y$ has a combined branch point and essential singularity at $y=y_{0}$ but regarded as a function of the real variable $y$, it has continuous derivatives of all orders there and is thus effectively completely regular.

The simplest application of Green's functions is to problems in which the solution of (1.1) is sought which satisfies the same homogeneous boundary 
conditions as the Green's function. The solution is given by

$$
\phi(x, y)=\iint G\left(x, y ; x_{0}, y_{0}\right) F\left(x_{0}, y_{0}\right) d x_{0} d y_{0},
$$

where the integration is taken over the whole of the relevant area. A somewhat non-rigorous but nevertheless very revealing interpretation of this equation is to say that since an arbitrary function $F(x, y)$ can be thought of as a continuous linear superposition of delta functions through the equation

$$
F(x, y)=\iint \delta\left(x-x_{0}\right) \delta\left(y-y_{0}\right) F\left(x_{0}, y_{0}\right) d x_{0} d y_{0},
$$

then the required solution is that same linear superposition of the solutions when given delta functions are on the right hand side. We note that the requirement of homogeneity in our boundary conditions is an essential one.

A far more subtle application of Green's functions is to the problem of finding the solution of (1.1) when non-homogeneous boundary conditions are given on the boundary. We can now take $F(x, y)$ to be zero without loss of generality since otherwise we would merely have to add a solution of the form (1.6). In order to proceed we must first define the adjoint boundary value problem. The adjoint operator $\tilde{L}$ is related to $L$ by the requirement that

$$
\psi L[\phi]-\phi \tilde{L}[\psi]=\frac{\partial P}{\partial x}+\frac{\partial Q}{\partial y},
$$

where $P, Q$ are functions of $\phi, \psi$ and their first derivatives with respect to $x$ and $y$. If now $\psi$ is set equal to $\widetilde{G}\left(x, y ; x_{0}, y_{0}\right)$ which is some Green's function for the adjoint operator $\tilde{L}$ and if (1.7) is integrated over the domain and Green's theorem applied,

$$
\phi\left(x_{0}, y_{0}\right)=\int_{C} Q d x-P d y,
$$

where $C$ is the boundary of the domain, $P, Q$ are functions of $\phi, \widetilde{G}$ and their first derivatives and the integration is taken in the positive sense. We have not yet specified any homogeneous boundary conditions for $\widetilde{G}$. This we do by first assuming temporarily that the given boundary conditions for $\phi$ on $C$ are homogeneous which will cause some of the terms in the curvilinear integral in (1.8) to vanish. Then we demand that $\widetilde{G}$ satisfy the minimum number of homogeneous boundary conditions on $C$ to make the right hand side of (1.8) vanish completely. Now, when non-homogeneous boundary conditions for $\phi$ are restored, $\phi\left(x_{0}, y_{0}\right)$ is given in terms of these known boundary values and the values of $G$ and its derivatives on $C$. The demands made on $\widetilde{G}$ cause it to vanish at just the appropriate places to annihilate any nonspecified data concerning $\phi$ on $C$. Up to this point the argument is somewhat academic as we have not related $\widetilde{G}$ to $G$ but now comes a striking simplification. If we set $\phi=G\left(x, y ; x_{1}, y_{1}\right)$ and $\psi=\widetilde{G}\left(x, y ; x_{0}, y_{0}\right)$ in (1.7) and integrate 
over the domain bounded by $C$, we find that

$$
G\left(x_{0}, y_{0} ; x_{1}, y_{1}\right)=\widetilde{G}\left(x_{1}, y_{1} ; x_{0}, y_{0}\right) \text {. }
$$

In other words, the adjoint Green's function $\widetilde{G}$ is obtained from the original by merely interchanging the active variables $x, y$ with the parameter variables $x_{0}, y_{0}$. If $\widetilde{G}=G$, the boundary value problem is self-adjoint and the Green's function is symmetric in the active and parameter variables. It is easily verified that the boundary value problem quoted earlier for Laplace's equation is self-adjoint but that this is not true of either of the other two. The differential operator itself is not self-adjoint for the diffusion equation and though the wave equation operator is self-adjoint, the initial value problem is not since the boundary conditions satisfied by $\tilde{G}$ are different from those satisfied by $G$. Accordingly, these Green's functions are not symmetric in the active and parameter variables as is indeed confirmed by equations (1.4) and (1.5). Some authors point out that there is a quasi-symmetry by introducing causality arguments relating to the direction of the time variable but while these physical arguments are helpful in some ways they are not in any way essential to the mathematical development of the subject. On the other hand, we must clarify what we mean by the domain round which we integrate. For Laplace's equation or any other elliptic equation, a closed domain bounded by a curve $C$ is the natural one for a properly posed problem, the data being given on $C$. In our example the domain is in fact a semi-infinite plane with $\phi \rightarrow 0$ at infinity being a boundary condition. For the wave equation and the diffusion equation, data are given on $y=0$. They may or may not be given on spatial boundaries as well but for simplicity we may assume that the string or bar is infinite in both directions. In any event, the aggregate of data-carrying curves will be an open curve. We therefore "close" the domain by drawing some line $y=Y$ where $Y$ is a constant supposed greater than any particular value of $y$ in which we may be interested and thereafter apply Green's identity to the area $-\infty<x<\infty, 0<y<Y$ or to the finite rectangle $x_{1}<x<x_{2}, 0<y<Y$ if spatial boundary conditions are given on $x=x_{1}$ and $x=x_{2}$. The adjoint Green's function $\widetilde{G}\left(x, y ; x_{0}, y_{0}\right)$ for the wave equation must then satisfy the wave equation and the boundary conditions $\widetilde{G}=\partial \widetilde{G} / \partial y=0$ on $y=Y$. This defines $G$ independently of $Y$ except in so far as we require $y_{0}<Y$ and, as demanded by the general theory, $\widetilde{G}$ is given by (1.4) with $x, y$ interchanged with $x_{0}, y_{0}$ respectively. A straightforward application of Green's identity then gives the familiar d'Alembert solution when $\phi$ and $\partial \phi / \partial y$ are specified on $y=0$. We omit the details.

\section{Riemann's method}

At first sight, Riemann's method appears to operate quite differently. We shall consider it applied to the equation

$$
L[\phi] \equiv \frac{\partial^{2} \phi}{\partial x^{2}}-\frac{\partial^{2} \phi}{\partial y^{2}}+2 g \frac{\partial \phi}{\partial x}-2 f \frac{\partial \phi}{\partial y}+c \phi=F(x, y),
$$


where $g, f$ and $c$ are functions of $x$ and $y$, this being the most general form of linear hyperbolic equation of the second order in two variables. If $\phi$ and $\partial \phi / \partial n$, the normal derivative, are given on a curve $\mathscr{L}$ whose slope is less than one in modulus (Fig. 1), then $\phi\left(x_{0}, y_{0}\right)=\frac{1}{2}(\phi R)_{A}+\frac{1}{2}(\phi R)_{B}+\frac{1}{2} \int_{A B}\left\{\left(R \frac{\partial \phi}{\partial y}-\phi \frac{\partial R}{\partial y}+2 f \phi R\right) d x\right.$
$\left.+\left(R \frac{\partial \phi}{\partial x}-\phi \frac{\partial R}{\partial x}+2 g \phi R\right) d y\right\}-\frac{1}{2} \iint_{P_{0} A B} R\left(x, y ; x_{0}, y_{0}\right) F(x, y) d x d y$

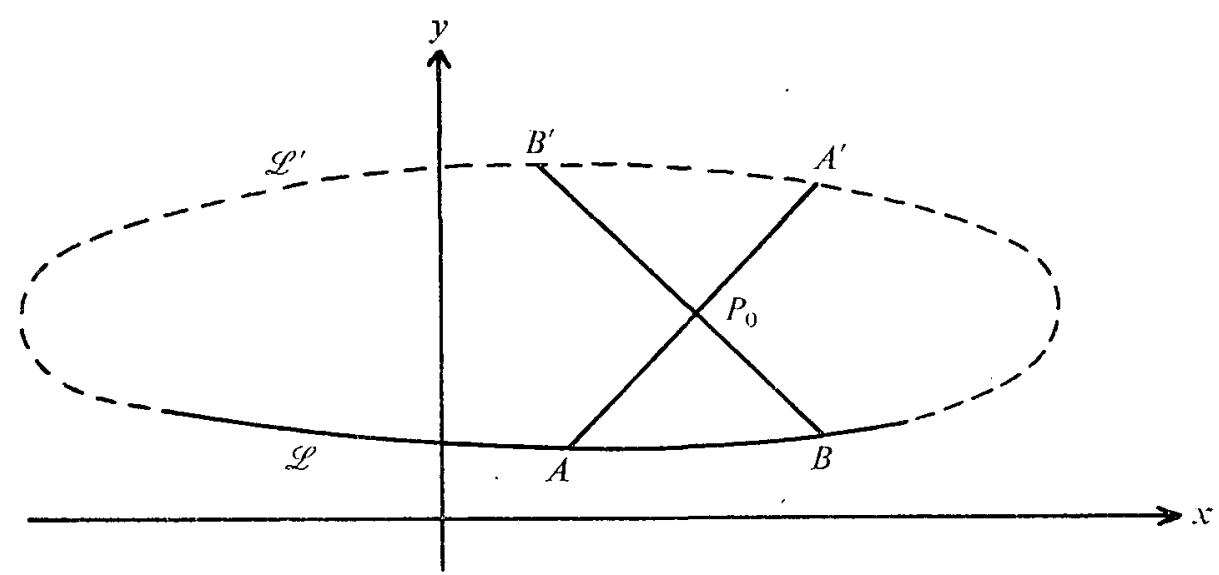

FIG. 1.

where $A, B$ are the points where the characteristics through $P_{0}\left(x_{0}, y_{0}\right)$ meet $\mathscr{L}$ and $R\left(x, y ; x_{0}, y_{0}\right)$ is the Riemann function. This function satisfies the following four conditions:

$$
\begin{aligned}
& \tilde{L}[R] \equiv \frac{\partial^{2} R}{\partial x^{2}}-\frac{\partial^{2} R}{\partial y^{2}}-2 \frac{\partial(g R)}{\partial x}+2 \frac{\partial(f R)}{\partial y}+c R=0 \\
& \frac{\partial R}{\partial x}+\frac{\partial R}{\partial y}=(g+f) R \text { on } y-x=y_{0}-x_{0} \\
& \frac{\partial R}{\partial x}-\frac{\partial R}{\partial y}=(g-f) R \text { on } y+x=y_{0}+x_{0}, \\
& R\left(x_{0}, y_{0} ; x_{0}, y_{0}\right)=1 .
\end{aligned}
$$

Let us now suppose that we wish to solve this boundary value problem by means of Green's functions. The appropriate Green's function $G\left(x, y ; x_{0}, y_{0}\right)$ is defined as the solution of

$$
L[G]=\delta\left(x-x_{0}\right) \delta\left(y-y_{0}\right)
$$

which vanishes with its normal derivative on $\mathscr{L}$. In order to obtain a closed curve $C$ we draw some curve $\mathscr{L}^{\prime}$, shown dotted in Fig. 1 , so that $C=\mathscr{L}+\mathscr{L}^{\prime}$. The adjoint Green's function $\tilde{G}$ now vanishes with its normal derivative on $\mathscr{L}^{\text {. }}$ 
and we have

$\tilde{G} L[\phi]-\phi \tilde{L}[\tilde{G}]=\frac{\partial}{\partial x}\left(\tilde{G} \frac{\partial \phi}{\partial x}-\phi \frac{\partial \tilde{G}}{\partial x}+2 g \phi \tilde{G}\right)-\frac{\partial}{\partial y}\left(\tilde{G} \frac{\partial \phi}{\partial y}-\phi \frac{\partial \tilde{G}}{\partial y}+2 f \phi \tilde{G}\right)$

which, when integrated over $D$, the interior of $C$, gives

$$
\begin{aligned}
\phi\left(x_{0}, y_{0}\right)=-\int_{C}\left\{\left(\widetilde{G} \frac{\partial \phi}{\partial y}-\phi \frac{\partial \widetilde{G}}{\partial y}\right.\right. & \left.+2 f \phi \widetilde{G}) d x+\left(\widetilde{G} \frac{\partial \phi}{\partial x}-\phi \frac{\partial \widetilde{G}}{\partial x}+2 g \phi \tilde{G}\right) d y\right\} \\
& +\iint_{D} \widetilde{G}\left(x, y ; x_{0}, y_{0}\right) F(x, y) d x d y .
\end{aligned}
$$

On the basis of what happened with the ordinary wave equation we would expect the Green's function $G$ to vanish except in the right angle $B^{\prime} P_{0} A^{\prime}$. If so, this means that $\widetilde{G}$ will vanish except in $P_{0} A B$. Thus in (2.5) $D$ can be replaced by $P_{0} A B$ in the double integral and the contour $C$ can be replaced (apparently) by $A B$ in the curvilinear integral. If now we consider the special case in which the homogeneous data $\phi=\partial \phi / \partial n=0$ are prescribed on $C$ and compare (2.1) with (2.6), then the line integrals vanish in each and we are led to assume that

$$
R\left(x, y ; x_{0}, y_{0}\right)=-2 \widetilde{G}\left(x, y ; x_{0}, y_{0}\right)
$$

in regions where $\widetilde{G}$ does not vanish. The whole argument can be confirmed and seen in a more spectacular fashion by using equation (2.1) to determine the Green's function $G\left(x, y ; x_{1}, y_{1}\right)$ for the operator $L$ with associated homogeneous boundary conditions on $\mathscr{L}$. It is obtained from (2.1) by setting $\phi=\partial \phi / \partial n=0$ on $A B$ and $F(x, y)=\delta\left(x-x_{1}\right) \delta\left(y-y_{1}\right)$. Then by the properties of the delta function

$$
\begin{aligned}
G\left(x_{0}, y_{0} ; x_{1}, y_{1}\right) & =-\frac{1}{2} R\left(x_{1}, y_{1} ; x_{0}, y_{0}\right) & \text { if }\left(x_{1}, y_{1}\right) \text { lies inside } P_{0} A B, \\
& =0 & \text { if }\left(x_{1}, y_{1}\right) \text { lies outside } P_{0} A B .
\end{aligned}
$$

By virtue of (1.9), it follows that $\widetilde{G}\left(x, y ; x_{0}, y_{0}\right)$, regarded as a function of $x$ and $y$, vanishes except in $P_{0} A B$ where it coincides with the Riemann function divided by a factor of -2 .

There remains one point to be cleared up. Equation (2.6) does not apparently reduce to equation (2.1) if $-\frac{1}{2} R$ is substituted for $\bar{G}$ in (2.6). There remain outstanding the first two terms $\frac{1}{2}(\phi R)_{A}$ and $\frac{1}{2}(\phi R)_{B}$ on the right hand side of (2.1). However, these arise in (2.6) also from the integration of the derivatives $\partial \widetilde{G} / \partial x$ and $\partial \widetilde{G} / \partial y$ over lines across which $\widetilde{G}$ itself is discontinuous. An elementary investigation shows that the residual terms are accounted for in exactly this way.

We have thus established a close connexion between the Green's function and the Riemann function. That they should be so closely related is at first sight surprising for two reasons. Firstly, the conditions which define the Riemann function are quite regular and, if regular properties of the coefficients $g, f$ and $c$ are assumed, will lead to a regular function, whereas the Green's function satisfies a non-homogeneous equation with a delta function, which 
we might fairly call a substantial singularity, on the right hand side. Secondly, the Riemann function is uniquely defined by the differential operator whereas, as we have seen, the Green's function has to be specified by homogeneous conditions on some boundary curve. The first discrepancy is accounted for by the fact that minus twice the adjoint Green's function coincides with the Riemann function only in the interior of a right angle. The Green's function vanishes outside this angle and the sharp discontinuity thus obtained is the direct result of the presence of the delta function on the right hand side of the equation which defines it. As for the second point, the particular right angle in which the adjoint Green's function is non-zero is determined by the position of the initial or data-carrying curve $\mathscr{L}$. The Green's function for hyperbolic equations is not nearly so sensitive to the location of the datacarrying curve as is that for elliptic equations and for a given point $P_{0}\left(x_{0}, y_{0}\right)$ it has only four distinct values depending upon whether $\mathscr{L}$ is above or below or to the left or right of $P_{0}$ (bearing in mind that the slope of $\mathscr{L}$ must nowhere be that of a characteristic, namely \pm 1 ). Closer examination will show that in the two of these cases where the slope of $\mathscr{L}$ exceeds one in modulus, $R=2 \tilde{G}$ in the region where $\widetilde{G} \not \equiv 0$ whereas in the other two cases (one of which we have demonstrated in detail) $R=-2 \widetilde{G}$ in this region.

\section{The Euler-Poisson equation}

Apart from unifying different methods of solving boundary value problems for hyperbolic differential equations, the above correspondence principle affords an additional method of finding Riemann functions. It also leads to some interesting questions regarding the domain of definition of these functions. We illustrate by considering the equation in the study of which Riemann devised his method. This is the equation which is now frequently called the Euler-Poisson equation, namely

$$
\frac{\partial^{2} \phi}{\partial X \partial Y}+\frac{N}{X+Y}\left(\frac{\partial \phi}{\partial X}+\frac{\partial \phi}{\partial Y}\right)=0
$$

or, in non-characteristic co-ordinates $x, y$ where $X=x+y, Y=x-y$,

$$
\frac{\hat{o}^{2} \phi}{\partial x^{2}}+\frac{2 N}{x} \frac{\partial \dot{\phi}}{\partial x}-\frac{\partial^{2} \phi}{\partial y^{2}}=0
$$

and we consider the domain to the right of the singular line $x=0$ only. It is sometimes useful to remember that this equation is the radially symmetric wave equation in a space of $2 N+1$ dimensions.

The Green's function satisfies

$$
\frac{\partial^{2} G}{\partial x^{2}}+\frac{2 N}{x} \frac{\partial G}{\partial x}-\frac{\hat{o}^{2} G}{\partial y^{2}}=\delta\left(x-x_{0}\right) \delta\left(y-y_{0}\right) \text {. }
$$

Let us now suppose that $x_{0}>0, y_{0}>0$ and demand that $G$ vanish with $\partial G / \partial y$ 
on $y=0$ and be finite on the singular line $x=0$. If both sides of (3.1) are multiplied by $x^{N+\frac{1}{2}} J_{N-\frac{1}{2}}(\rho x)$ and integrated with respect to $x$ from 0 to $\infty$, then after some manipulation

where

$$
\frac{d^{2} \bar{G}}{d y^{2}}+\rho^{2} \bar{G}=-x_{0}^{N+\frac{1}{2}} J_{N-\frac{1}{2}}\left(\rho x_{0}\right) \delta\left(y-y_{0}\right),
$$

$$
\bar{G}\left(y ; x_{0}, y_{0} ; \rho\right)=\int_{0}^{\infty} G\left(x, y ; x_{0}, y_{0}\right) x^{N+\frac{1}{2}} J_{N-\frac{1}{2}}(\rho x) d x .
$$

The solution of (3.2) which vanishes with its derivative at $y=0$ is

$$
\bar{G}\left(y ; x_{0}, y_{0} ; \rho\right)=-\rho^{-1} x_{0}^{N+\frac{1}{2}} J_{N-\frac{1}{2}}\left(\rho x_{0}\right) H\left(y-y_{0}\right) \sin \rho\left(y-y_{0}\right)
$$

and by Hankel's inversion theorem

$G\left(x, y ; x_{0}, y_{0}\right)=-\frac{x_{0}^{N+\frac{1}{2}}}{x^{N-\frac{1}{2}}} H\left(y-y_{0}\right) \int_{0}^{\infty} \sin \rho\left(y-y_{0}\right) J_{N-\frac{1}{2}}\left(\rho x_{0}\right) J_{N-\frac{1}{2}}(\rho x) d \rho$.

Reference to an appropriate table of infinite integrals shows that near $\left(x_{0}, y_{0}\right)$ this expression is zero except in the right angle formed by the characteristics through $\left(x_{0}, y_{0}\right)$ which point in the direction of increasing $y$ where it is given by

$$
G\left(x, y ; x_{0}, y_{0}\right)=-\frac{1}{2}\left(\frac{x_{0}}{x}\right)^{N} P_{N-1}\left\{\frac{x^{2}+x_{0}^{2}-\left(y-y_{0}\right)^{2}}{2 x x_{0}}\right\} .
$$

Interchange of $(x, y)$ with $\left(x_{0}, y_{0}\right)$ and multiplication by -2 gives

$$
R\left(x, y ; x_{0}, y_{0}\right)=\left(\frac{x}{x_{0}}\right)^{N} P_{N-1}\left\{\frac{x^{2}+x_{0}^{2}-\left(y-y_{0}\right)^{2}}{2 x x_{0}}\right\}
$$

in the entire neighbourhood of $\left(x_{0}, y_{0}\right)$, an expression given, for example, by Copson (1).

We have used the word neighbourhood and we must now come to define this concept more carefully. If the functions $g, f$ and $c$ are analytic in the immediate neighbourhood of $P_{0}\left(x_{0}, y_{0}\right)$, then it is not difficult to see that the Riemann function is an analytic function of $x$ and $y$ (that is, of the complex variables $x$ and $y$ separately) near $\left(x_{0}, y_{0}\right)$. This follows from the boundary conditions (2.3)-(2.5) which define the Riemann function, these specifying a regular characteristic boundary value problem for the hyperbolic equation (2.2). Recognition of this fact enables us to define in the following way the region in which $R\left(x, y ; x_{0}, y_{0}\right)$ itself will be analytic. A point $P(x, y)$ will lie in this region if the characteristic rectangle, of which $P P_{0}$ is a diagonal, is one in which $g, f$ and $c$ are regular and analytic. For the Euler-Poisson equation under consideration, this means the region in which $y-x<x_{0}+y_{0}$, $y+x>y_{0}-x_{0}$ and $x>0$ simultaneously or the region between the dotted lines 
and to the right of the $y$-axis in Fig. 2. There is now no difficulty in defining the Riemann function from the Green's function. The Green's function is non-zero only in a right angle with vertex at $P_{0}$ but after interchanging $(x, y)$ with $\left(x_{0}, y_{0}\right)$ and multiplying (in this case) by -2 we continue analytically the function so obtained to define the Riemann function throughout the region just indicated. The continuation is in this case automatic and immediate because the representation (3.4) is analytic throughout the region considered.

In the remainder of the half-plane $x>0$ (we regard the singular line $x=0$ as a barrier across which no passage is allowed) different considerations apply.

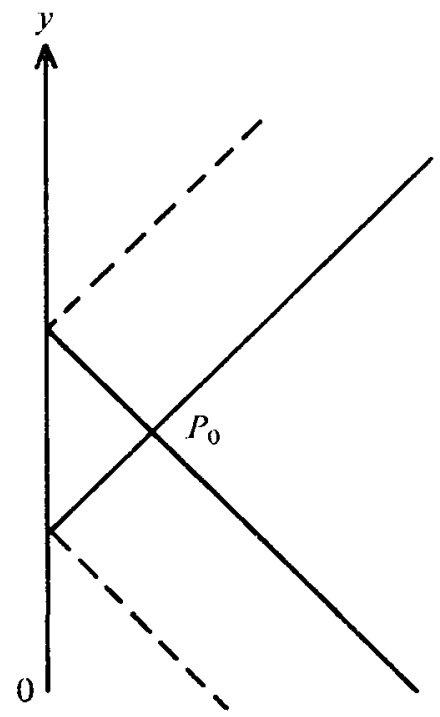

Fig. 2.

Here our point of view differs somewhat from that adopted by Copson (1) who divided the half-plane $x>0$ into the six different regions indicated in Fig. 2 and found an expression for the Riemann function in each of the six regions. In the four regions with $\left(x_{0}, y_{0}\right)$ on their boundary, $R$ is given by (3.4) whereas in the remaining two regions Copson gives the expression which would be obtained by ignoring the Heaviside function and evaluating (3.3), interchanging $(x, y)$ with $\left(x_{0}, y_{0}\right)$ and multiplying by -2 , namely (in our notation)

$$
R\left(x, y ; x_{0}, y_{0}\right)=\frac{2 \sin N \pi}{\pi}\left(\frac{x}{x_{0}}\right)^{N} Q_{N-1}\left\{\frac{\left(y-y_{0}\right)^{2}-x^{2}-x_{0}^{2}}{2 x x_{0}}\right\} .
$$

It is now relevant, however, to ask the question, in what sense can this be said to be the Riemann function? If this function is regarded as being defined solely by (2.2)-(2.5), then these remaining two regions do not appear to come within the ambit of this definition, it being impossible to form a characteristic rectangle with the line joining $(x, y)$ to $\left(x_{0}, y_{0}\right)$ as diagonal. It should also be 
observed that (3.5) is in no way the continuation of (3.4) in any normally accepted sense. Let us take the case $N=1$ for simplicity. Then according to (1), $R=x / x_{0}$. between the dotted lines and zero above and below them and is therefore not even continuous across the dotted lines. It would appear that the precise manner of defining the Riemann function is crucial. We prefer to take the simple view that $R$ is defined only by the solution of the characteristic boundary value problem (2.2)-(2.5) and that it is not defined at any point $P$ if this point is such that no characteristic rectangle with $P P_{0}$ as diagonal lies inside the domain of operation of the equation. According to this definition, this means that for the Euler-Poisson equation $R$ is defined by (3.4) in the region between the dotted lines and is undefined elsewhere.

It should be emphasised that by contrast it is entirely meaningful to speak of the Green's function throughout the entire region $x>0$. This function $G\left(x, y ; x_{0}, y_{0}\right)$, which satisfies (3.1) together with the boundary conditions $G=\partial G / \partial y=0$ on $y=0$ and a condition of finiteness on the singular line $x=0$, is given by (3.3) for all $x>0$ and all $y$. When $N=1$ we can identify this with the solution to a physical problem of air vibrations. If $\phi$ is the velocity potential, the problem is that of air at rest prior to $y=y_{0}$ at which time there is a sudden radially symmetric disturbance on an infinitely thin shell $x=x_{0}$ ( $x$ being the radial co-ordinate). The disturbance moves both out and in and after it reaches the origin it is reflected outwards. The fact that $G=0$ after the reflection is a manifestation of the Huygens' principle. It is easily seen from (3.4) that $G=0$ for any integer $N$ after such a reflection. This again is a Huygens' principle effect since when $N$ is a positive integer, the Euler-Poisson equation as defined here is the radially symmetric wave equation for an odd number $2 N+1$ of space dimensions.

\section{REFERENCE}

(1) E. T. Copson, On the Riemann-Green function, Arch. Rat. Mech. Anal. 1 (1958), 324-348.

VICTORIA UNIVERSITY OF WELLINGTON

NeW ZEALAND 